\title{
Radiation in low doses reduces life span of Daphnia magna*
}

\author{
E. Sarapultseva ${ }^{1}$, J. Malina $^{1}$ and A. Gorski ${ }^{2}$ \\ ${ }^{1}$ Institute of Nuclear Power Engineering of the National Research Nuclear University \\ "MEPhl", Obninsk, Studgorodok, 1, Biology Dept., Russian Federation \\ ${ }^{2}$ National Epidemiologic Registry of the Medical Radiological Research Center, Obninsk, \\ Zhukov St., 8, Russian Federation
}

\begin{abstract}
The effects of low-dose $\gamma$-irradiation on the crustacean Daphnia magna have been analyzed. Given that the $\mathrm{LD}_{50}$ for this species is approximately $100 \mathrm{~Gy}$, one-day-old daphnids were exposed to 10,100 and $1000 \mathrm{mGy}$ of acute $\gamma$-rays. The survival of irradiated animals was evaluated over the maximum lifespan of 90 days. Increased mortality among irradiated at doses of 100 and $1000 \mathrm{mGy}$ daphnids was found. Our data also showed that the observed decrease in survival of irradiated animals was attributed to their early aging, thus providing an important evidence for the effects of low-dose irradiation on early senescence. Overall, the results of our studies have shown that low-dose irradiation can result in a significant reduction in life span of exposed daphnids. As the majority of irradiated Daphnia showed reduced viability regardless the dose, our data therefore imply the presence of a threshold of dose of low-LET exposure capable of impairing the viability of this species. We have also found that parental irradiation significantly affected the survival of first-generation offspring, the magnitude of which cannot be attributed to the segregation of radiation-induced deleterious mutations. Potential implications of the results of our studies for the risk assessment of low-dose exposure have been discussed.
\end{abstract}

\section{INTRODUCTION}

Issuer of low doses radiation effects on life span of animals and humans are currently far from resolved. First of all, this is due to the requirement of large volumes of observations to obtain sufficient statistical power of the experiment. This can be achieved by increasing population size or time of observation, which in turn is associated with technical and financial difficulties.

Our earlier observation of the increase of mortality crustaceans Daphnia magna after acute $\gamma$-irradiation may have particular interest in connection with issuer of the effects of low doses radiation on life span $[1,2]$.

Daphnids represent a very useful experimental model, which allows the very efficient and quick analysis of many aspects of non-targeted effects of ionising radiation, including radiation-induced genomic instability manifesting in the directly exposed animals and their offspring. The life span of these animals is relatively short and seldom exceeds 10-11 weeks; they are also characterised by quite short durations of embryonic development (3-4 days) and gestation (5-8 days). Most importantly, Daphnia magna 'enjoy' parthenogenetic reproduction, which quickly allows establishing genetically identical strains of these animals.

Doses of 10, 100 and $1000 \mathrm{mGy}$ were selected for this study from a group of doses previously tested for daphnids $(10,100,1000 \mathrm{mGy}$ and 20,100, 250, $600 \mathrm{~Gy}$ [1]). The aim of this study is to analyze the dose dependence of life span and mortality risk of population daphnids after acute $\gamma$-irradiation in low doses.

\footnotetext{
*The current investigation is a part of a research project of the Federal Target Program "Research and scientific-pedagogical personnel Innovative Russia" in 2009-2013 years.
} 


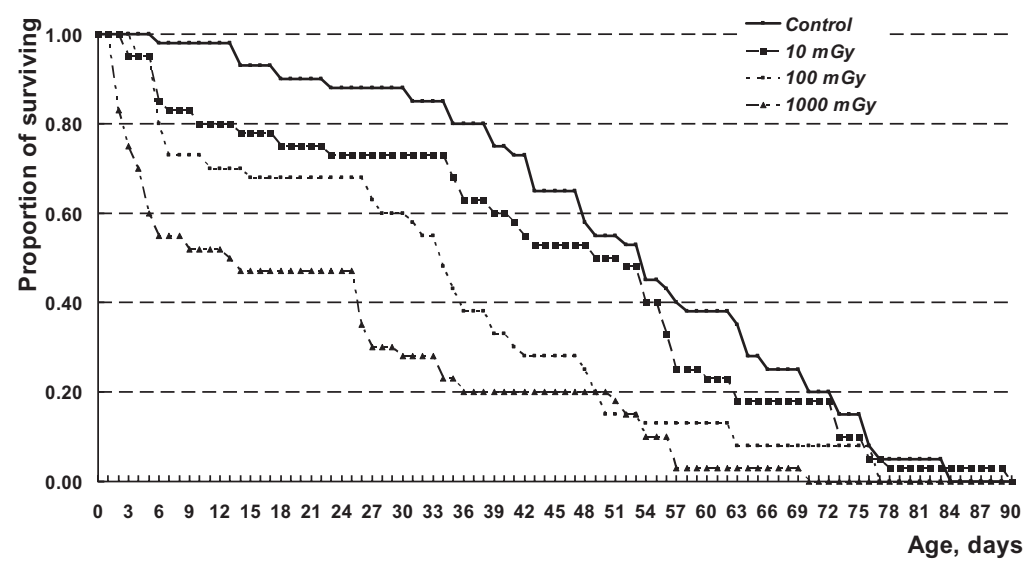

Figure 1. Dynamics of the survival of the daphnids' populations in the control and after irradiated at doses of 10 , 100 and $1000 \mathrm{mGy}$ until their death. Original number of populations (40 in each sample) was taken as a unit.

Table 1. The average life span and risk of mortality in the control and the irradiated at doses of 10, 100 and $1000 \mathrm{mGy}$ populations of daphnids.

\begin{tabular}{|l|c|c|c|c|}
\hline Dosa (mGy) & Control & 10 & 100 & 1000 \\
\hline Period of observations (days) & 60 & 60 & 60 & 60 \\
\hline Average life span (days) & 47.2 & 40.0 & 30.9 & 21.5 \\
\hline The risk of mortality & 0.62 & 0.75 & 0.88 & 0.85 \\
\hline Risk difference of mortality in the exposed and control populations & - & 0.12 & 0.25 & 0.38 \\
\hline Bottom 95\% confidence limit of risk difference & - & -0.08 & 0.06 & 0.18 \\
\hline Top 95\% confidence limit of risk difference & - & 0.33 & 0.44 & 0.57 \\
\hline
\end{tabular}

\section{RESULTS}

It is known that radiation doses of $250 \ldots 600 \mathrm{~Gy}$ lead to mortality of crustaceans in the early period after exposure $\left(\mathrm{LD}_{100 / 16} \ldots \mathrm{LD}_{100 / 4}\right.$, respectively) [1]. At the same time in the study of survival of daphnids exposed in much lower doses, we also found a lethal effect. It, however, has had a qualitatively different nature. Fig. 1 shows the survival curves of daphnids' population irradiated at doses of 10, 100 and 1000 mGy. Populations were observed until their death.

It is seen that mortality of the irradiated populations is more intense than in the control. It is interesting to note that sharp decline in survival occurs in 5-6 days, at the gestation period when eggs formed. We can assume that the mortality of organisms which are most sensitive to radiation occurs in the early periods after irradiation at doses 100 times lower than the $\mathrm{LD}_{50}$.

Analysis of survival curves of daphnids in the control and irradiated at a dose of $100 \mathrm{mGy}$, showed that the confidence limit intersect at some point in time (20,60 days) and do not intersect at other periods (40 days). Therefore, using only this criterion is not sufficient to assess of the effect of low radiation doses. Comparison of the difference or the ratio of risk of mortality in the irradiated and control populations over the observation period, when the number of individuals in populations greater than or equal to 5 (according to [3]), allowed us to estimate the effectiveness of low doses of radiation.

In Table 1 the average life span, the risks of mortality, the difference between the risk of mortality and confidence limits (bottom and top) in the control and irradiated populations of daphnids are represented.

Table 1 shows a decrease of average life span and an increase of mortality risk in the irradiated population with the increasing of irradiation dose. The effect of exposure, namely significant excess of risk of mortality in the exposed population relative to control, was observed at doses of 100 and 


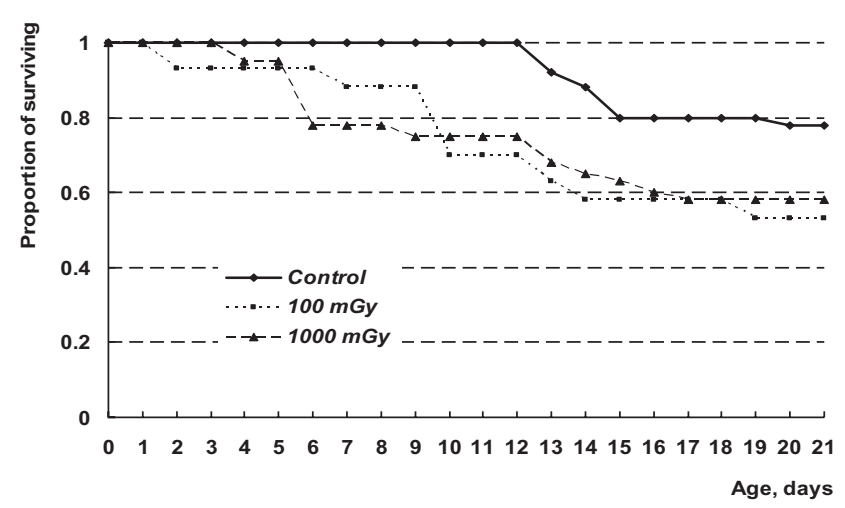

Figure 2. Dynamics of the survival of daphnids in the $F_{1}$ generation in the control and non-irradiated populations derived from irradiated at doses of 100 and 1000 mGy daphnids-parents. Original number of populations (40 in each sample) was taken as a unit.

Table 2. The average life span and risk of mortality in the control and the first generation of non-irradiated daphnids which were born from irradiated at doses of 100 and $1000 \mathrm{mGy}$ daphnids-parents.

\begin{tabular}{|l|c|c|c|}
\hline Doses of $\gamma$-irradiated of daphnids-parents, (mGy) & Control & 100 & 1000 \\
\hline Time (period) of observations (days) & 30 & 30 & 30 \\
\hline Average life span (days) & 26.5 & 20.2 & 21.2 \\
\hline The risk of mortality & 0.32 & 0.52 & 0.45 \\
\hline $\begin{array}{l}\text { Risk difference of mortality in the exposed } \\
\text { and control populations }\end{array}$ & - & 0.20 & 0.12 \\
\hline Bottom 95 (90)\% confidence limit of risk difference & - & $-0.02(0.02)$ & $-0.09(-0.05)$ \\
\hline Top 95 (90)\% confidence limit of risk difference & - & $0.42(0.38)$ & $0.34(0.30)$ \\
\hline
\end{tabular}

$1000 \mathrm{mGy}$. The positive value of $95 \%$ confidence limit for the risk difference was displayed. It means that the effect is statistically significant. Let's compare, using the presented approach, the risk of mortality in exposed populations. So a difference between the risk of mortality, irradiated at doses of 10 and $1000 \mathrm{mGy}$ populations is statistically significant, at doses of $10-100 \mathrm{mGy}$ and $100-1000 \mathrm{mGy}-$ it is not statistically significant.

Now let's consider the results of observation of the non-irradiated first-generation offspring. Fig. 2 shows the dynamics of survival in the control and in the population of theirs broods which were obtained from irradiated at doses of 100 and 1000 mGy daphnids-parents.

At fig. 2 you can see a similar pattern as at the Fig. 1. In the populations of irradiated daphnids, as in the non-irradiated generation, the number of fatal events has increased more substantially than in the control.

Table 2 shows the average life span of the non-irradiated offspring of $F_{1}$, the risk of mortality, the difference in mortality risk and confidence limits (bottom and top) in the control and in the nonirradiated populations of daphnids, which were born from irradiated at doses of 100 and $1000 \mathrm{mGy}$ daphnids-parents.

As shown in Table 2, the non-irradiated populations of daphnids, obtained from the first brood of irradiated at doses of 100 and 1000 mGy daphnids-parents have a shorter life span than the control of the first generation. Life span of non-irradiated specimens of the first generation, as well as directly exposed animals is reduced by an average of 1.5 times. The risk of mortality in the first generation of daphnids which were born from irradiated at doses of 100 and 1000 mGy parents increased non-significantly at $\mathrm{p} \leq 0.05$. The negative values of bottom $95 \%$ confidence limit for the risk difference evidenced about 
it. However, the confidence limit of risk difference for the dose of $100 \mathrm{mGy}$ becomes positive at $90 \%$ validity bottom value and remains statistically significant for a group of $1000 \mathrm{mGy}$.

\section{CONCLUSION}

Thus, in the present study, the lowest dose causing a statistically significant effect of reducing the survival rate (1.5 times) and life span (35\%) of daphnids is a dose of $100 \mathrm{mGy}$ of acuter $\gamma$-radiation. The doses of 50,100,500 and $1000 \mathrm{mGy}$ were the lowest doses which causing an effect according to reduced survival of spleen cells of rainbow trout and the activity of enzymes of oxidative phosphorylation [4], survival of mice' endothelia cells [5], cytogenetic damage on plants [6] and molecular-genetic changes in generations of mice [7] appropriately. Apparently, a threshold dose below which the somatic and/or genetic effect could not be induced is in the range of doses 50-1000 mGy.

The risk difference of mortality in populations of crustacean, irradiated at doses of 100 and $1000 \mathrm{mGy}$, was not statistically significant. Perhaps the loss of value connected with the decrease in statistical power due to a reduction of the analyzed period of observation. This fact requires additional research. However, a doses-independent effect in the diapason of low radiation doses was found earlier in a change of physiological activity in populations of aquatic protozoa ciliates Spirostomum [8, 9]. The data of present study also showed that the observed decrease in survival of irradiated animals was attributed to their early aging, thus providing an important evidence for the effects of low-dose irradiation on early senescence. Reduced life span of irradiated at $400 \mathrm{mGy}$ mice were found in a study [10]. All of these convince us in the reality of the observation effects of low-dose radiation exposures.

The study of non-irradiated daphnids' populations showed that first-generation offspring inherit of dose-independent damage induced in daphnids parental exposure at low doses. Some loss of statistical significance of biological effects in the offspring may be associated with a short period of observation ( 30 days). On the other hand it can be assumed that exposure to daphnids-parents at a dose of $1000 \mathrm{mGy}$ removes from the population most sensitive individuals (see Figure 1). Perhaps this fact leads to a decreased risk of mortality in the first non-irradiated generation due to the formation of those populations from radio-resistant individuals. However, analysis of the data showed that the overall risk of mortality among non-irradiated offspring of irradiated at doses 100 and $1000 \mathrm{mGy}$ daphnids is in average 1.5 times higher than in control, and their life span is less on $20 \%$.

Transgenerational decrease of survival and reduce of life span among the first generation offspring of irradiated parents may be associated with "non-targeted" mechanism of this phenomenon. Unusual features of the damage that we found in this and other studies [2, 9], namely: the effectiveness of low-dose, dose-independence and long-term manifestations of damage, similar to the phenotype of the effects which were described in the collective group of "non-targeted" effects. Our data on the inheritance of damage in the first generation daphnids offer the opportunity to transfer at organismic level of genomic instability arising in irradiated parents.

The practical significance of the data is important for solving problems of radiation protection of biota. It seems rather high because of the universality of the observed phenomenon and extremely low for species of effective threshold of exposure.

\section{Acknowledgments}

The authors would like to thanks Dr. Geraskin for critical reading of the manuscript.

\section{References}

[1] Sarapultseva E.I., Malina J.J. Radiat. Biology. Radioecology, 49, 1 (2009) 82-84 [in rus].

[2] Sarapultseva E.I., Bychkovskaya I.B. Int. J. Low Radiation, 7, 1 (2010) 1-9. 
[3] Modern Epidemiology (K. Rothman \& S. Greenland, Ed., Publ. Lippincott Williamce \& Wilkins, 1998, pp. 254-320.

[4] O’Dowd C., Mothersill C.E., Cairns M.T. et al, Rad. Research, 171 (2009) 464-473.

[5] Kirik O.V., Stepanov R.P., Fedortseva R.F. et al, Int. J. Low Radiation, 7, 3, (2010) 208-216.

[6] Geras'kin S.A, Oudalova A.A., Kim J.K. et al, Rad. Environ. Biophys., 46 (2007) 31-41.

[7] Barber R.C., Hardwick R.J., Shanks M.E. et al, Mut. Res., 664 (2009) 6-12.

[8] Sarapultseva E.I. Radiat. Biology. Radioecology, 48, 3 (2008) 346-348 [in rus].

[9] Sarapultseva E.I., Bychkovskaya I.B. "Unusual damaging effects of low radiation. Model experiments with protozoa and invertebrates", $3^{\text {d }}$ European IRPA-2010, Helsinki, June 18-24, 2010, Prima Oy, Ed. (NSFS, Helsinki), pp. 1-7.

[10] Oghiso Y., Tanaka S., Tanaka I.B. III, Sato F., Int. J. Low Rad., 5, 1 (2008) 55-59. 